\title{
The developing bleb: effect of topical antiprostaglandins on the outcome of glaucoma fistulising surgery
}

\author{
CLIVE MIGDAL AND ROGER HITCHINGS
}

From the Glaucoma Unit, Moorfields Eye Hospital, High Holborn, London WC1V 7AN

SUMMARY A prospective trial of topical antiprostaglandins versus placebo in the treatment of postoperative fistulising surgery followed up for 12 months is described. It is concluded that antiprostaglandins have little place in the management of these cases. A photographic record of evolution of the fistulising bleb is described, together with a scoring system devised for the evolution of the successful fistulising bleb. A comparison is made between appearance and function of the fistulising bleb based on the use of the water drinking test and tonography together with intraocular pressure as tests of bleb function. It is concluded that the appearance of the established bleb alone is an insufficient guide to function, particularly if further surgery-for example, cataract extraction-is required.

To understand the factors which contribute to the formation of a functioning drainage bleb after glaucoma fistulising surgery it is essential, firstly, to define the various stages that occur during the course of development of a bleb, and thereafter to ascertain whether there are any factors which might predict poor function. It should then be possible to investigate methods by which the bleb function could possibly be improved.

The trabeculectomy operation was designed to allow aqueous to drain through a protected fistula, thereby minimising the possible hazards of transconjunctival leakage or infection. The difference between success or failure of the procedure may well depend to a large extent on the inflammatory healing process which follows surgery. This is seen histologically in the form of extensive fibroblastic proliferation in tissue removed when refashioning adherent blebs. ${ }^{1}$ Methods of reducing this inflammation might well therefore improve functional results.

This prospective clinical study was set up with 2 purposes in mind: firstly, carefully to observe and record developing blebs, and secondly to ascertain the effects of topical antiprostaglandins on developing blebs. Antiprostaglandins are known inhibitors of

Correspondence to Mr R. A. Hitchings, FRCS.
Table 1 Inflammation grading

1. Globe congestion

(a) Conjunctival vessels dilated

(i) None

(ii) Region of conjunctival incision

(iii) $360^{\circ}$

(b) Episcleral vessels dilated

(i) None

(ii) Region of conjunctival incision

(iii) $360^{\circ}$

2. Anterior chamber

(a) Cells

(i) None

(ii) $1+(1-5$ Cells/fine slit beam)

(iii) $2+(10-20$ Cells/fine slit beam)

(iv) $3+$ (More than 20 cells)

(v) $4+$ (Hypopyon)

(b) Exudate

(i) None

(ii) Strand

(iii) Coagulum

(c) Iris

(i) Dilated vessels yes/no

(ii) Posterior synechiae yes/no

(d) Blood

(i) Free floating RBC

(ii) Clot on iris

(iii) Hyphaema

(e) Flare

(i) Nil

(ii) Just visible

(iii) Marked 


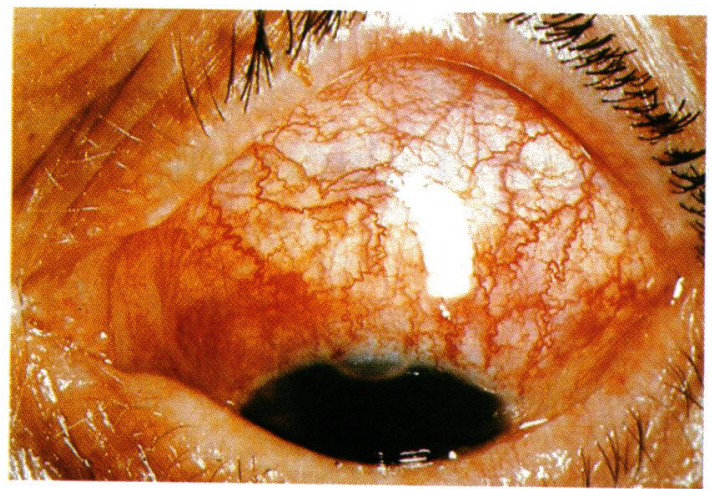

Fig. 1 Grade I bleb: absent.

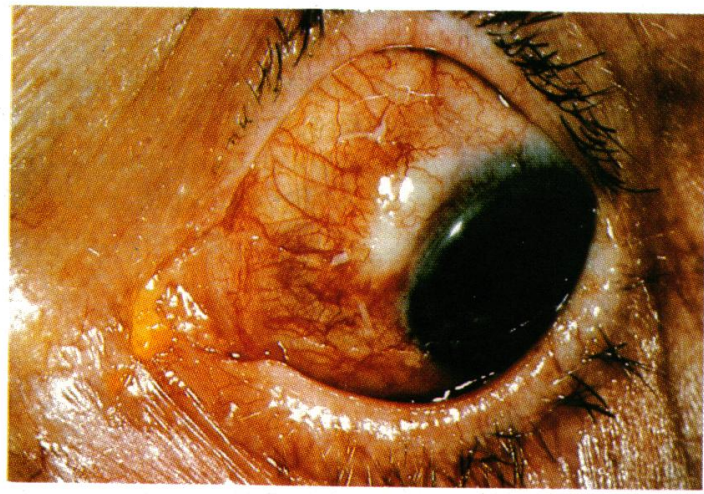

Fig. 3 Grade III bleb: Pale elevated area within engorged conjunctiva.

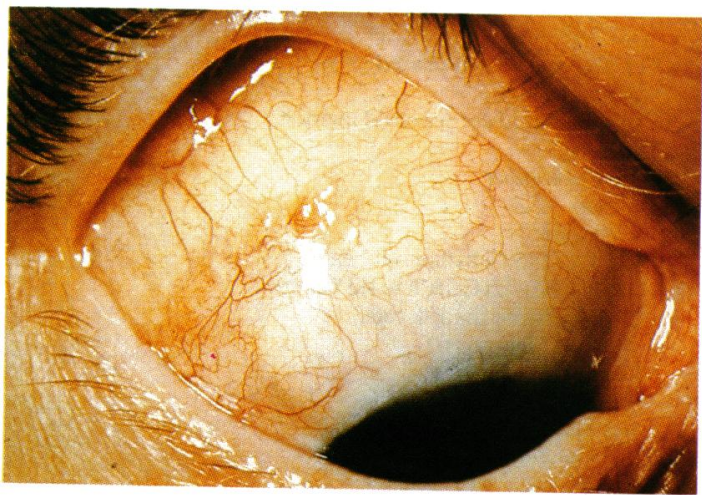

Fig. 5 Grade V bleb: Pale and diffusely elevated conjunctiva.

inflammation and as such might be expected to play a dominant role in the development of the normal fistulising bleb. The effect of antiprostaglandins in the eye has been studied both in vivo and in vitro. ${ }^{2-11}$

This paper links the inflammatory changes,

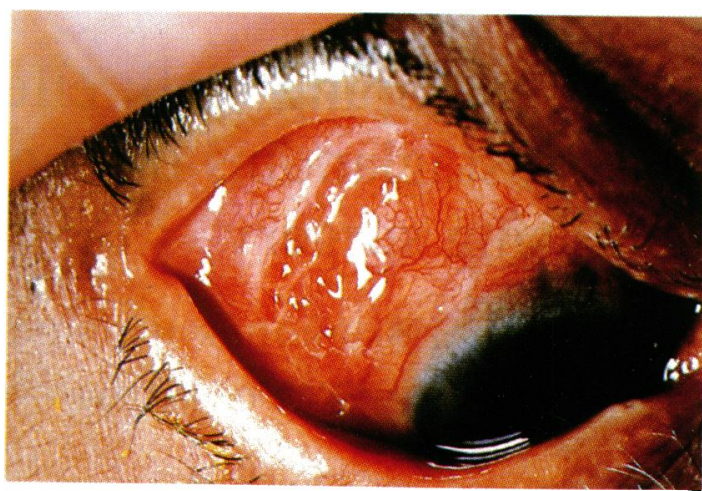

Fig. 2 Grade II bleb: Elevated engorged conjunctiva.

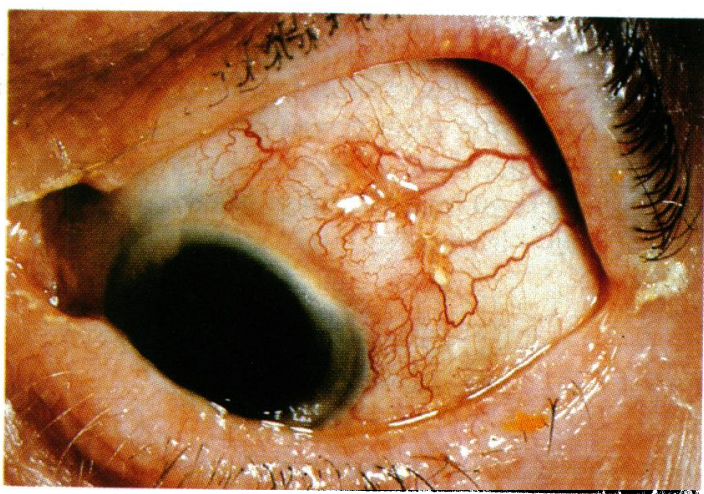

Fig. $4^{\prime}$ Grade IV bleb: Residual conjunctival vessel engorgement around the suture line.

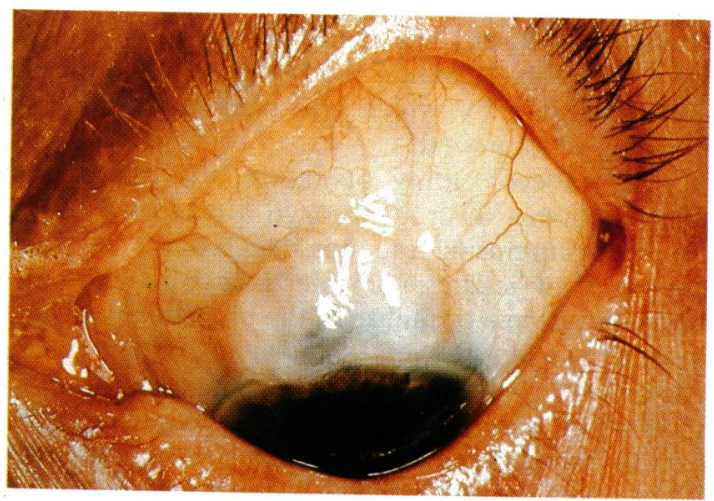

Fig. 6 Grade VI bleb: Cystic conjunctival elevation.

morphology, and functional aspects of developing blebs in 2 groups of patients over a follow-up period of 12 months. One group of patients received topical antiprostaglandins both pre- and postoperatively, while the second received a placebo. 
Table 2 Assessment of bleb formation

\begin{tabular}{ll}
\hline Drainage bleb \\
\hline 1. Absent \\
2. Elevated engorged conjunctiva \\
3. Pale elevated area within engorged conjunctiva \\
4. Residual conjunctival vessel engorgement around suture line \\
5. Pale and diffusely elevated conjunctiva \\
6. Cystic conjunctival elevation \\
\hline
\end{tabular}

\section{Material and methods}

The subjects of this study were 30 patients who underwent standard filtering surgery for chronic simple glaucoma, as described by Cairns. ${ }^{12}$ Every effort was made to conform to a standard surgical technique, with the scleral flaps being fashioned to an equal thickness. The eyes were randomly assigned to one of 2 groups designated to receive either topical antiprostaglandins (indomethacin $0.5 \%$ ) or a placebo (its vehicle, sesame oil).

Patients who had a past history of eye surgery, uveitis, or treatment with systemic steroids were excluded from the trial, while a record was made of any systemic diseases likely to affect the blood aqueous barrier - such as diabetes, haematological disorders, haemorrhage, and inflammatory forms of arthritis. All current medical treatment was documented; the $\mathrm{C}$ value and highest recorded intraocular pressure were charted preoperatively.

The trial drug (either gutt. indomethacin or the placebo) was given 60 minutes and 30 minutes preoperatively with the premedication.

Postoperatively the trial drug was given thrice daily, gutt. Predsol (prednisolone) 1\% 4 times daily, and gutt. atropin. $1 \%$ twice daily, all for one month.

The response of the eye to glaucoma surgery was assessed by measuring the vision, intraocular pressure, and outflow facility. The inflammatory

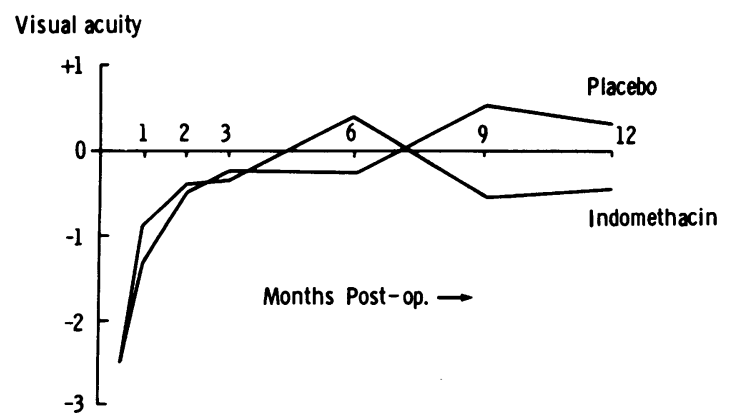

Fig. 7 Mean postoperative visual acuity, recorded as lines gained or lost on Snellen chart by comparison with the preoperative visual acuity. response in the anterior chamber and the conjunctiva was graded (Table 1), and a reproducible grading system was devised to assess bleb formation and evolution (Table 2).

Colour photographs were obtained of every possible variety of developing blebs and these were numbered 1 to 6 (Figs. 1-6) according to reducing degrees of inflammation. At each clinic visit the patient's bleb was compared and matched with the relevant photograph and the 'bleb score' thus allocated.

Findings were recorded at weekly intervals for the first month, then monthly for 2 months, with review at 6,9, and 12 months. (A small number of patients attended out of phase in the later stages of follow-up, but this did not appear significantly to alter the statistics.) A minimum follow-up of 12 months was obtained, but some patients were followed up for up to 2 years.

To assess functional reserve ${ }^{13} 19$ patients ( 24 eyes) were subjected to a water drinking test on completion of the assessment. After measurement of the intraocular pressure 1 litre of water was consumed over a 5-minute period, and several measurements of the intraocular pressure made at 20,40 , and 60 minutes thereafter. Control of intraocular pressure was compared with the earlier $\mathrm{C}$ values.

\section{Results}

Fourteen eyes received indomethacin (group A) and 16 the placebo (group B). One group A patient was lost to long-term follow-up.

The ages of the patients ranged from 36 to 73 years. There were no associated systemic illnesses deemed likely to effect the bleb outcome.

\section{FUNCTION}

The effect on best corrected vision has been set up in Fig. 7, that on intraocular pressure in Fig. 8, and on outflow facility in Fig. 9. There are a number of specific points worth noting.

Vision was reduced immediately postoperatively in both groups. The acuity at 12 months had returned to the preoperative level in 13 eyes, was reduced in 8 , but improved in a further 8 . The mean final vision improved above the preoperative mean in the placebo group.

Although 4 patients had had intermittent intraocular pressures recorded above $21 \mathrm{mmHg}$ during the first 3 months of the follow-up period, all intraocular pressures had returned to normal without further surgery by 9 months with the exception of one indomethacin-treated patient. It was concluded that an early intermittent increase in IOP was not necessarily an index of failure. 
Fig. 8 Intraocular pressure ( $\mathrm{mmHg}$ ).

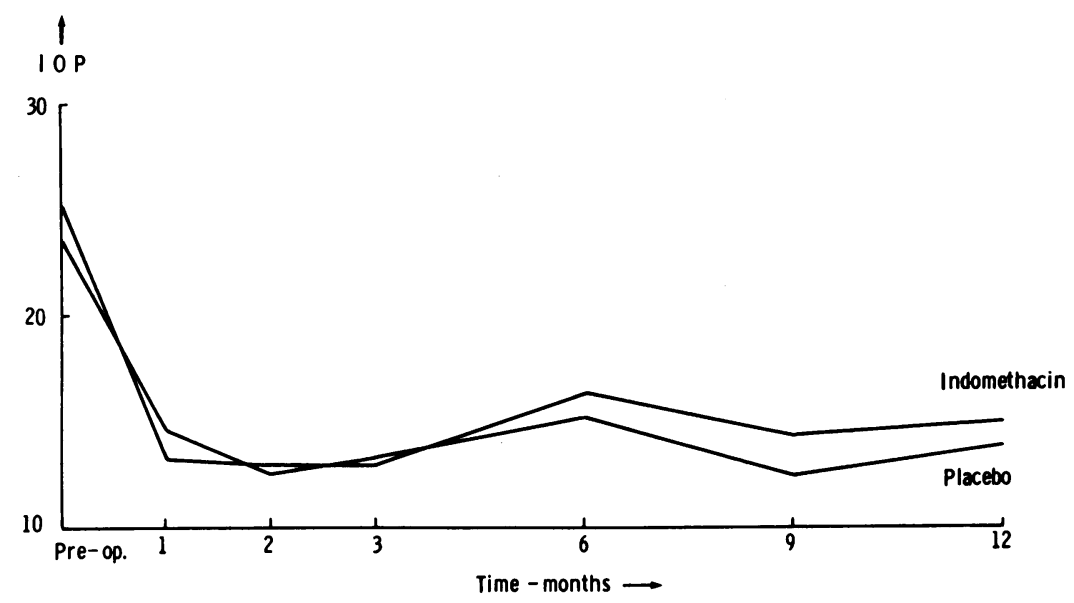

A low $\mathrm{C}$ value in the postoperative period correlated well with malfunctioning of the drainage bleb in those patients with raised intraocular pressure.

During the water drinking test, performed at the end of the study, only 2 patients showed poor control of IOP, one from group A and one from group B. In both these cases the $C$ values at 3 months had been on the low side, being $0 \cdot 10$ and $0 \cdot 12$ respectively.

By our criteria the intraocular inflammation, which was an important aspect of the immediate postoperative phase, had resolved by 3 months. There

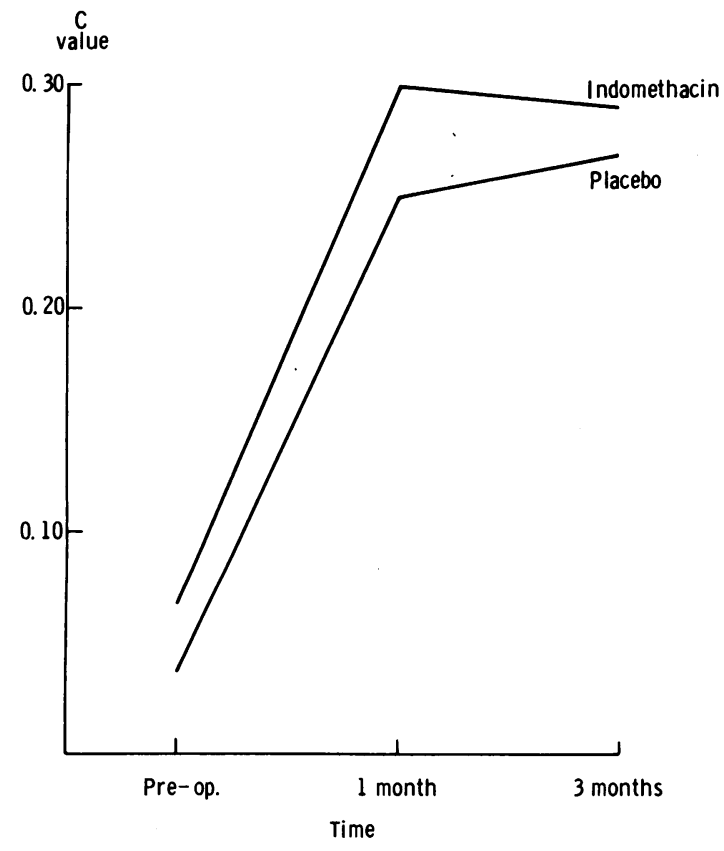

Fig. 9 Outflow facility. was no significant difference between the 2 groups of patients.

\section{BLEB MORPHOLOGY}

The bleb score of individual eyes having been assessed at each visit, the mean bleb score was calculated for the 2 groups at each visit. The method of scoring used appeared to work very well, with a linear improvement up the scale from grade 1 to 5 or 6 in most cases. A low bleb score correlated well with the inflammatory changes and poor bleb function in those blebs with recorded raised intraocular pressure.

While bleb formation evolved in a more or less similar fashion in the 2 groups for the first 2 months, statistical analysis of the scores at the 2- and 3-month assessments showed a significantly higher score in the control patients than in those receiving indomethacin $(p<0.02$, Mann-Whitney U test). This difference had disappeared by the end of the study. These findings are summarised in Fig. 10. It is worth noting, however, that there was a higher incidence of cystic blebs in the control group $(6 / 16$ versus $2 / 13)$.

\section{Discussion}

Our preliminary report ${ }^{14}$ describing the effect of topical antiprostaglandins on developing blebs studied over a 3-month period showed that patients treated with topical indomethacin drops developed a greater cellular reaction in the anterior chamber in the immediate postoperative period and a greater inflammatory response around the bleb at 2 and 3 months postoperatively.

This increased inflammatory response outside the eye persisted for months and was associated with higher intraocular pressures and lower $C$ values in some cases. Inflammatory activity inside the eye was short lived and of questionable significance. Topical 


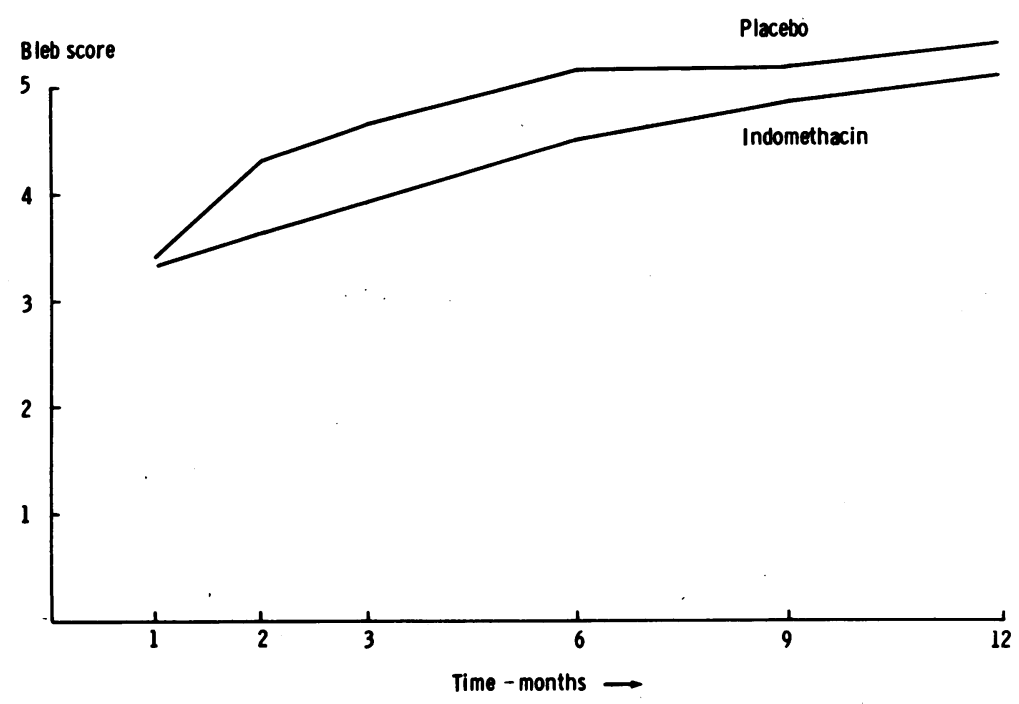

Fig. 10 Mean bleb score

Predsol (prednisolone) forte given q.i.d. to both groups of patients did not completely prevent postoperative inflammation.

Pharmacological testing of the drops after the conclusion of the trial failed to reveal any factor other than the indomethacin itself to account for the difference seen in the 2 groups of patients. However, recent investigations into the role of antiprostaglandins have shown a potentiation of conjunctival hyperaemia and polymorphonucleocyte response in the rabbit model, ${ }^{15}$ which could be explained by the differential breakdown of the unsaturated fatty acids released at surgery via the lipoxygenase pathway to HETE (which potentiates cellular mobility and chemotaxis at the site of injury, seen clinically as fibrosis $\left.{ }^{14}\right)$.

Despite the fact that the recorded acuity may be the same or better than that preoperatively, patients may complain of a rather nonspecific visual disturbance. With the exception of one patient in the control group, no significant lens opacities or nuclear sclerosis were detected to account for these symptoms. It is possible that the cessation of topical antiglaucoma treatment may be responsible, or that posterior synechiae, in restricting pupillary movement, may influence the ability to focus.

Notwithstanding a significant difference in the first 3 months, the mean bleb scores in the 2 groups of patients became similar by the 9-month stage and remained thus, though there was a higher incidence of cystic blebs in the control group. Although this difference may reflect greater early postoperative inflammation, the small numbers do not allow definite conclusions to be drawn. The difference between the 2 groups was also reflected in the fact that most eyes in the indomethacin treated group had lower scores, a feature not only restricted to the failures, and this again emphasises the delayed evolution of the bleb.

The bleb scoring system used in this study proved to be a reproducible grading system for describing the morphological appearance of developing fistulising blebs. The use of photographs worked well, enabling the progress of individual patients to be documented and compared.

Our bleb scoring system has identified appearances of the bleb at different times in eyes that were later shown to have had successful surgery. Conversely, should the appearance of the drainage bleb not match the score appropriate for the time after surgery, then this could be an indication of failure at that operation. As failure is likely to result from an excessive fibrovascular response round the bleb, treatment should be directed to reducing this process. At present such treatment would include the thorough application of topical steroids and incision of the bleb ${ }^{16}$ but not, on our findings, the administration of topical antiprostaglandins.

A distinction needs to be drawn between morphological appearance of the bleb and function. The water drinking test showed considerable reserve in bleb function in most of our cases. However, 2 eyes showed a rise in intraocular pressure. Tonography (another test of functional reserve) showed low C values for these eyes, though the final bleb scores were not noticeably different between eyes with and without positive water drinking tests. This wide range of bleb function, despite the similar late appearance, may go some way to account for failure of the 'successful' fistulising operation in some eyes that have subsequent cataract surgery. Tonography and/or 
the water drinking test could be useful predictors of bleb function in the preoperative assessment of such cases. If poor bleb function is discovered, then revision of the bleb or refiltration should be carried out at the time of cataract surgery.

\section{CONCLUSION}

From this prospective trial of topical antiprostaglandins versus placebo for eyes undergoing fistulising surgery it is concluded that antiprostaglandins have little place in the management of these cases. A reproducible photographic method of grading developing blebs has been devised, and it has been shown that there may be little relationship between the appearance and functional reserve of the fistulising bleb. The significance of this finding for such eyes undergoing later cataract surgery is discussed.

We are grateful to Kay Mills for secretarial and administrative assistance, and to Biddy Finch who conducted the water drinking tests.

C. S. Migdal was supported by a grant from the Frost Foundation.

\section{References}

1 Addicks EA, Quigley HA, Green WR. The histology of glaucoma filtering blebs. Invest Ophthalmol Visual Sci 1981; 20 (ARVO suppl): 29.

2 Chavis RM, Vygantas CM, Vygantas A. Experimental inhibition of prostaglandin-like inflammatory response after cryotherapy. Am J Ophthalmol 1976; 82: 310-2.

3 Unger WG, Bass MS. Prostaglandin and nerve-mediated response of the rabbit eye to argon laser irradiation of the iris. Ophthalmologica 1977; 175: 153-8.
4 Mochizuki M, Sawa M, Masuda K. Topical indomethacin in intracapsular extraction of senile cataract. Jpn J Ophthalmol 1977; 21: 215-26.

5 Whitelocke RAF, Eakins KE, Bennett A. Acute anterior uveitis and prostaglandins. Proc R Soc Med 1973; 66: 429-34.

6 Masuda K, Izawa Y, Mishima S. Prostaglańdins and glaucomatocyclitic crisis. Jpn J Ophthalmol 1975; 19: 368-75.

7 Miyake K, Sugiyama S, Norimatsu I, Osawa T. Prevention of cystoid macular edema after lens extraction by topical indomethacin. III. Radioimmunoassay measurement of prostaglandins in the aqueous during and after lens extraction procedures. Albrecht Von Graefes Arch Klin Ophthalmol 1978; 209: 83-8.

8 Klein RM, Katzin HM, Yanuzzi LA. The effect of indomethacin pretreatment on aphakic cystoid macular edema. Am J Ophthalmol 1979; 87: 487-9.

9 Miyake K, Sugiyama S, Norimatsu I, Ozawa T. Aqueous prostaglandins in persistent cystoid macular edema secondary to vitreous incarceration: effects of pars plana vitrectomy. $J p n J$ Ophthalmol 1980; 24: 335-45.

10 Yannuzzi LA, Klein RM, Wallyn RH, Cohen N, Katz I. Ineffectiveness of indomethacin in the treatment of chronic cystoid macular edema. Am J Ophthalmol 1977; 84: 517-9.

11 Cooper CA, Bergamini MVW, Leopold IH. Use of flurbiprofen to inhibit corneal neovascularization. Arch Ophthalmol 1980; 98: 1102-5.

12 Cairns JE. Trabeculectomy. Preliminary report of a new method. Am J Ophthalmol 1968; 66: 673-9.

13 Kronfeld PC. The mechanism of filtering operations. Trans Pac Coast Otoophthal Med Soc 1949; 30: 23-40.

14 Migdal C, Hitchings R. Effect of antiprostaglandins on glaucoma filteration surgery. Trans Ophthalmol Soc UK 1982; 102: 129-32.

15 Kulkarni PS, Bhattacherjee P, Eakins KE, Srinivasan BD. Antiinflammatory effects of betamethasone phosphate, dexamethasone phosphate and indomethacin on rabbit ocular inflammation induced by bovine serum albumin. Curr Eye Res 1981; 1: 43-7.

16 Cohen JS, Shaffer RN, Hetherington J, Hoskins D. Revision of filtration surgery. Arch Ophthalmol 1977; 95: 1612-5. 